\title{
串联的铜、钯催化偶联-环化合成二芳胺基芴
}

\author{
蔡良珍萄茾刘涛平陶晓春*
}

(华东理工大学化学与分子工程学院 上海 200237)

\begin{abstract}
摘要 发展了一种由串联的铜、钯金属催化偶联-环化合成二芳胺基荡类衍生物的新方法. 取代的碘苯与甲基和溴原子 互为邻位的苯胺在铜催化下反应生成溴代甲基三苯胺, 然后与邻氯苯基硼酸在钯催化下发生 Suzuki 偶联反应, 最后在 NHC-Pd 催化下发生 $\mathrm{C}\left(\mathrm{sp}^{3}\right)-\mathrm{H}$ 键活化-环化反应. 方便地合成了 2 位，尤其是 3 位取代的二芳胺基荡类化合物，且均获 得了不错的收率. 所有化合物均通过了 ${ }^{1} \mathrm{H}$ NMR, ${ }^{13} \mathrm{C}$ NMR, HRMS, IR, 紫外和荧光等手段的表征. 对其中两个具有代 表性的二芳胺基芴的结构运用二维核磁共振技术进行了解析.
\end{abstract}

关键词 二芳胺基芴; $\mathrm{C}-\mathrm{H}$ 活化; 铜催化; 钯催化

\section{Synthesis of Diarylaminofluorenes through Tandem Copper and Palladium Catalyzed Coupling-Cyclizing Reactions}

\author{
Cai, Liangzhen Lin, Hui Liu, Taoping Tao, Xiaochun*
}

(School of Chemistry and Molecular Engineering, East China University of Science and Technology, Shanghai 200237)

\begin{abstract}
A convenient method of tandem copper and palladium catalyzed coupling-cyclizing reactions has been developed for the preparation of diarylaminofluorenes. Methylbromotriphenylamines prepared by the copper-catalyzed reaction of substituted iodobenzenes and methylbromoanilines, coupled with chlorophenylboronic acid under palladium catalyst to obtain the chlorophenylmethyltriphenylamines $(\mathbf{V})$. A series of diarylaminofluorenes can be generated in good yields through palladium-catalyzed activation of benzylic $\mathrm{C}\left(\mathrm{sp}^{3}\right)-\mathrm{H}$ bond of compound $\mathbf{V}$. It was found that the 2-diarylaminofluorenes or 3-diarylaminofluorenes could be efficiently got. These compounds were characterized by ${ }^{1} \mathrm{H} N M R,{ }^{13} \mathrm{C}$ NMR, HRMS, IR and UV-vis.

Keywords diarylaminofluorene; $\mathrm{C}-\mathrm{H}$ activation; copper-catalyzed; palladium-catalyzed
\end{abstract}

芴类化合物具有刚性平面的联苯结构，能产生较宽 的能带隙、较高的发光效率以及良好的光学与化学稳定 性, 被广泛应用于有机薄膜场效晶体管 ${ }^{[1]}$ 、光致变色材

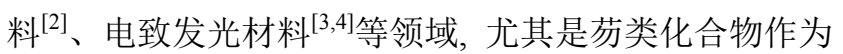
蓝光材料引起人们的兴趣 ${ }^{[5]}$.

经研究发现, 目前芴类光电材料存在长波发射以及 较差的空穴注入能力 ${ }^{[6,7]}$ 的问题. 因此不断地对其结构 进行修饰, 提高材料的光电性能, 是目前研究的重点 ${ }^{[8]}$. 将二芳胺基引入芴环结构中可以有效降低材料的电离 势, 提高材料的空穴传输能力 ${ }^{[9]}$. 另一方面, 被应用于 高效染料敏化太阳能电池(DSSCs) 中的有机染料分子, 大多具有一个 $\pi$ 共轭体作为连接给体基团(Donor)与受
体基团 (Acceptor) 的桥梁，以此形成 “ $\mathrm{D}-\pi-\mathrm{A}$ ” 的结 构 ${ }^{[10 \sim 12]}$. Agostina 等 ${ }^{[14]}$ 发现, 在芴的 3,6 位引入二芳胺基 给体基团(D), 它与 9 位的受体基团(A)能够通过中间的 芴环 ( $\pi$ 共轭体)有效地进行分子内电荷转移, 形成 $D-\pi-A$ 结构以提高材料的光电转换效率. 但 3,6 位取代的二芳 胺基苆类化合物合成难度非常大，通常以 9,10-菲醌为 原料, 首先在 3,6 位进行澳化, 然后在高锰酸钾的碱性 条件下脱羰, 再利用 Wolff-Kishner-黄鸣龙反应或硼氢 化物还原为澳代芴，随后进行 Buchwald-Hartwig 胺化反

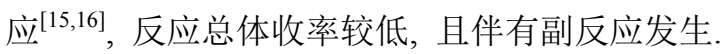

根据传统的方法，以荡为原料，通过澳化、硝化、 酰化等手段在苆环上引入取代基进行结构修饰，必然会

*E-mail: xctao@ecust.edu.cn

Received March 26, 2015; revised April 14, 2015; published online April 27, 2015.

Project supported by the Specialized Research Fund for the Doctoral Program of Higher Education (No. 20120074120008) and the Shanghai Key Laboratory of Molecular Catalysts and Innovative Materials (Fudan University, No. 2012MCIMKF01).

高等学校博士学科点专项科研基金(No. 20120074120008)和上海市分子催化和功能材料重点实验室(复旦大学, No. 201MCIMKF01)资助项目. 
受到芴环亲电反应规律的限制, 取代基的位置被限定在 芴环的 2 或 7 位上, 包括二芳胺基的引入亦是如此. 关 于其他 3 或 6 位取代反应的报道非常少 ${ }^{[14]}$. 我们设想, 以取代碘苯和不同结构的溴代甲基苯胺为原料, 先合成 具有二芳胺基的联苯类化合物, 再通过 $\mathrm{C}\left(\mathrm{sp}^{3}\right)-\mathrm{H}$ 键活 化一环化反应来构建芴环. 通过这种方法可以方便地、选 择性地合成 2 位，尤其是 3 位取代的芴类化合物(Scheme 1).

\section{1 结果与讨论}

\section{1 二芳胺基芴类化合物的合成}

\subsection{1 铜催化 III-A 和 III-B 的合成}

卤代芳烃与取代的苯胺反应生成三苯胺类化合物, 通常可以在铜或者钯金属催化下发生 ${ }^{[17]}$. 我们分别以3溴-4-甲基苯胺(I-A)和 3-甲基-4-溴苯胺(I-B)与碘苯(II) 进行反应, 发现当用钯与膦配体组合催化时, 反应比较 乱, 有所需要的三芳胺产物生成, 但也有其他副反应发 生, 可能是含溴底物所引起的. 基于此, 我们也做了笁 选, 发现当用铜金属作催化剂时, 碘化物活性要高于溴 化物, 这样便可以抑制含溴底物所引起的副反应. 最终 确定以 1,10 -菲啰啉为配体, 以氯化亚铜为催化剂, 在氢 氧化钾的存在下 ${ }^{[18]}$, 甲苯中加热回流 $24 \mathrm{~h}$, 发生
Ullmann 反应，选择性地得到含溴三苯胺类化合物 III-A 和 III-B，分离产率可达到 $80 \%$ 以上，见 Scheme 1 .

\subsection{2 钯催化 V-A 和 V-B 的合成}

钯催化卤代芳烃与苯基硼酸发生 Suzuki 偶联有许 许多多的反应体系 ${ }^{[19]}$ ，对于不同取代基的卤代芳烃与 苯基嗍酸除了正常的联苯类产物生成之外，还会有一些 副反应发生, 因此选择合适的反应体系是很重要的. 我 们参考了 Tao 等 ${ }^{[20]}$ 以异丙醇为溶剂、 $\mathrm{K}_{2} \mathrm{CO}_{3}$ 为碱的反 应体系, 以 $\mathrm{PdCl}_{2}\left(\mathrm{PPh}_{3}\right)_{2}$ 代替 $\mathrm{PdCl}_{2}$ 为催化剂, 用溴代甲 基三苯胺 $(\mathrm{III})$ 与邻氯苯嶰酸 $(\mathrm{IV})$ 反应, 高收率、高选择 性地得到了含氯苯基取代的三苯胺类化合物 V-A 和 V-B，见 Scheme 1 和表 $1,{ }^{1} \mathrm{H}$ NMR、 ${ }^{13} \mathrm{C}$ NMR 和 HRMS 均证明了这些化合物结构的正确性.

\subsection{3 钯催化 $\mathrm{C}\left(\mathrm{sp}^{3}\right)-\mathrm{H}$ 键活化一环化合成 VI-A 和 VI-B}

过渡金属催化活化 $\mathrm{C}-\mathrm{H}$ 键构建碳碳键能有效提高 合成效率，减少废料的产生，因此引起人们的注意. 目 前的研究主要集中在芳环 $\mathrm{C}\left(\mathrm{sp}^{2}\right)-\mathrm{H}$ 键的活化反应 ${ }^{[21,22]}$. 对于更具挑战性的 $\mathrm{C}\left(\mathrm{sp}^{3}\right)-\mathrm{H}$ 键的活化反应研究较少. $\mathrm{Hu}$ 和 $\mathrm{Liu}$ 等 ${ }^{[23,24]}$ 使用 $\mathrm{Pd}(\mathrm{OAc})_{2}$ 与大位阻的膦配体组合, 催化活化 2-甲基-2'-氯代联苯中的茮位碳氢键构建芴环.

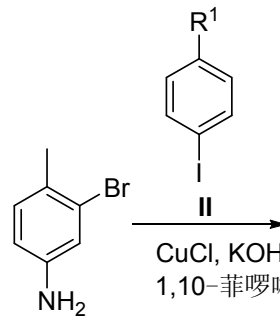

I-A

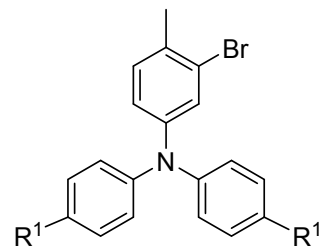

III-Aa: $\mathrm{R}^{1}=\mathrm{H}$ III-Ab: $\mathbf{R}^{1}=\mathrm{CH}_{3}$

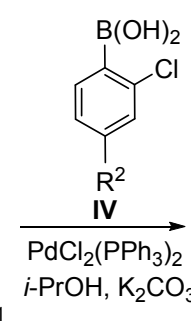

$$
\text { V-Aa: } R^{1}=H, R^{2}=H
$$$$
\text { V-Ab: } R^{1}=H, R^{2}=\mathrm{CH}_{3}
$$$$
\text { V-Ac: } \mathrm{R}^{1}=\mathrm{CH}_{3}, \mathrm{R}^{2}=\mathrm{H}
$$$$
\text { V-Ad: } \mathrm{R}^{1}=\mathrm{CH}_{3}, \mathrm{R}^{2}=\mathrm{CH}_{3}
$$

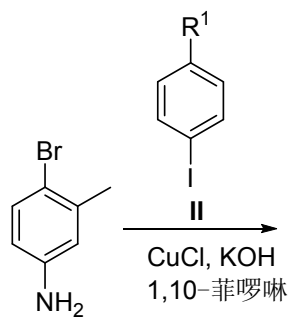

I-B

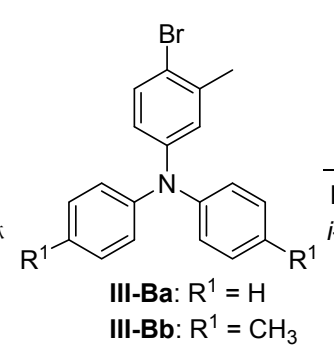

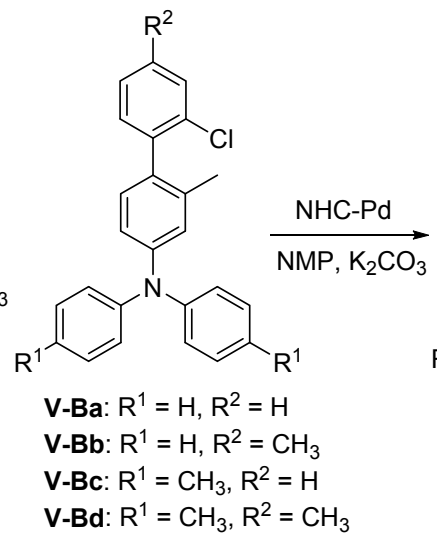
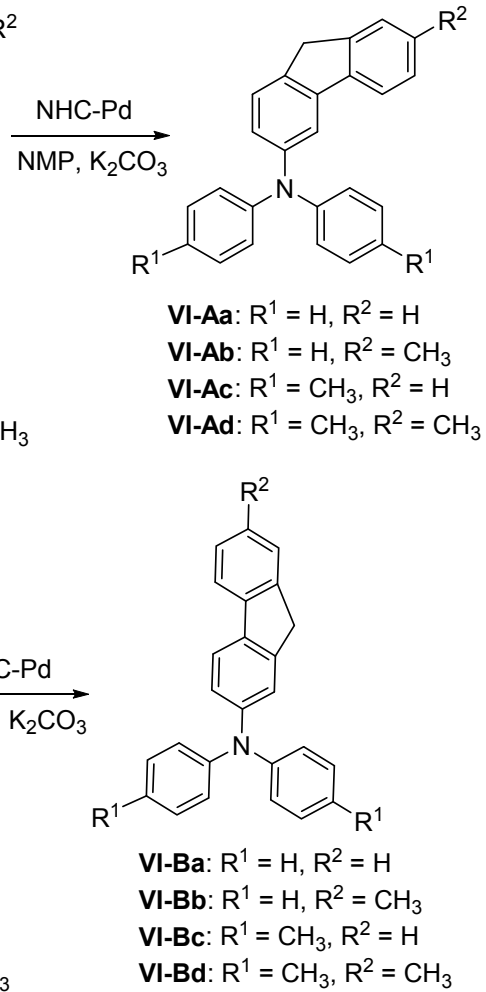

图式 1 二芳胺基芴的合成路线

Scheme 1 Synthetic routes of diarylaminofluorenes 
表 1 化合物 V-A、V-B 的合成 ${ }^{a}$

Table 1 Synthesis of V-A and V-B

\begin{tabular}{ccccc}
\hline Entry & $\mathrm{R}^{1}$ & $\mathrm{R}^{2}$ & 产物 $^{\text {产率 }^{b} / \%}$ \\
\hline 1 & $\mathrm{H}$ & $\mathrm{H}$ & $\mathbf{V}$-Aa & 94.1 \\
2 & $\mathrm{H}$ & $\mathrm{CH}_{3}$ & $\mathbf{V}$-Ab & 96.5 \\
3 & $\mathrm{CH}_{3}$ & $\mathrm{H}$ & $\mathbf{V}$-Ac & 92.7 \\
4 & $\mathrm{CH}_{3}$ & $\mathrm{CH}_{3}$ & $\mathbf{V}$-Ad & 96.1 \\
5 & $\mathrm{H}$ & $\mathrm{H}$ & $\mathbf{V}-\mathbf{B a}$ & 96.5 \\
6 & $\mathrm{H}$ & $\mathrm{CH}_{3}$ & $\mathbf{V}-\mathbf{B b}$ & 92.6 \\
7 & $\mathrm{CH}_{3}$ & $\mathrm{H}$ & $\mathbf{V}-\mathbf{B c}$ & 94.4 \\
8 & $\mathrm{CH}_{3}$ & $\mathrm{CH}_{3}$ & $\mathbf{V}-\mathbf{B d}$ & 93.7 \\
\hline
\end{tabular}

${ }^{a}$ Reaction conditions: III-A/III-B (1.0 mmol), IV (1.2 mmol), $\mathrm{Pd}\left(\mathrm{PPh}_{3}\right)_{2} \mathrm{Cl}_{2}$ $(0.01 \mathrm{mmol}), \mathrm{K}_{2} \mathrm{CO}_{3}(2.0 \mathrm{mmol})$ were mixed in $3 \mathrm{~mL}$ of refluxing isopropanol for $6 \mathrm{~h}$ under nitrogen; ${ }^{b}$ Isolated yield.

我们首先试验了这类体系, 但反应情况并不理想, 推测 可能是三芳胺的存在不利于三价膦配体的作用. 氮杂环 卡宾(NHC)是近十几年发展起来的一类新型的过渡金属 配体, 既具有膦配体的作用, 又有不同于膦配体的一些 特性 ${ }^{[25}$-27]. 它通常以易得到的、稳定的咪唑鈆氯盐来使 用, 反应时在碱的作用下当场生产氮杂环卡宾与金属配 位而起作用. $\mathrm{Wu}$ 等 ${ }^{[28]}$ 报道用醋酸钯与 1,3 -双 $(2,6$-二异丙 基苯基)咪唑鎓氯盐( $\mathrm{IPr} \cdot \mathrm{HCl}$ )组合催化 2-卤代-2'-甲基联 苯类化合物发生 $\mathrm{C}-\mathrm{H}$ 键活化并合环得到荡类化合物. 我们选用这个催化体系, 在碱的存在下，在 $N$-甲基吡咯 烷酮中, 化合物 V-A 或 V-B 发生 C-H 键活化环化反应, 分别得到 3-二芳胺基芴(VI-A)与 2-二芳胺基芴(VI-B), 分离产率可达到 $80 \%$ 左右(见 Scheme 1 与表 2). 该步反 应的可能机理 ${ }^{[29]}$ 见 Scheme 2. 首先 $\mathrm{Pd}^{0}$ 与化合物 $\mathbf{V}$ 的 $\mathrm{C}-\mathrm{Cl}$ 键进行氧化加成生成中间体 1 ; 然后 $\mathrm{CO}_{3}{ }^{2-}$ 取代 $\mathrm{Cl}$ 与 $\mathrm{Pd}$ 配位生成中间体 2 , 并掏取苄位碳上的一个质 子, 形成六元钯杂环中间体 3; 最后碱解离、还原消除得

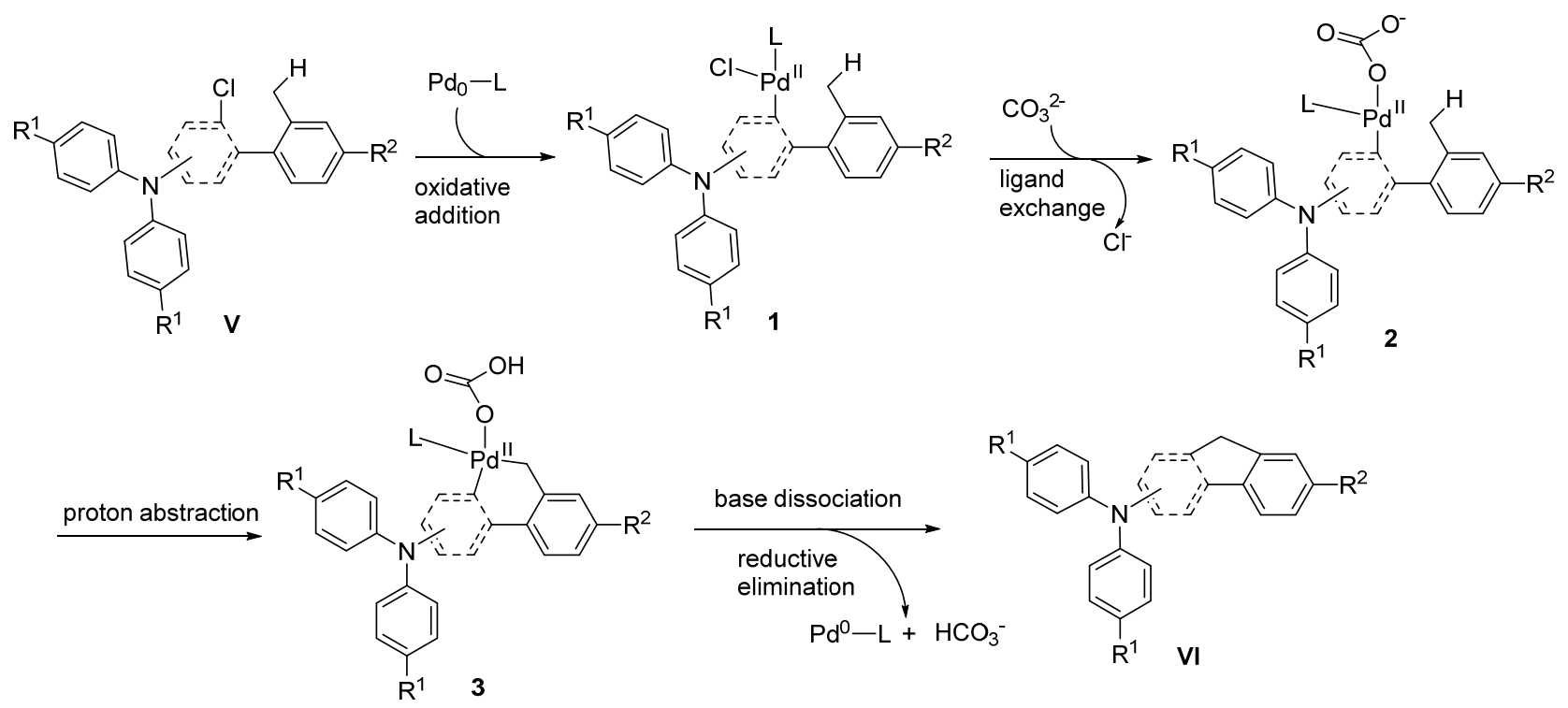

图式 2 钯催化环化反应的可能机理

Scheme 2 Probable mechanism of palladium catalyzed cyclizing reaction
到产物 VI, $\mathrm{Pd}^{0}$ 进入催化循环.

表 2 VI-A/VI-B 的合成 ${ }^{a}$

Table 2 Synthesis of VI-A and VI-B

\begin{tabular}{ccccc}
\hline Entry & $\mathrm{R}^{1}$ & $\mathrm{R}^{2}$ & 产物 & 产率 $^{b} \%$ \\
\hline 1 & $\mathrm{H}$ & $\mathrm{H}$ & VI-Aa & 83.4 \\
2 & $\mathrm{H}$ & $\mathrm{CH}_{3}$ & VI-Ab & 84.5 \\
3 & $\mathrm{CH}_{3}$ & $\mathrm{H}$ & VI-Ac & 82.9 \\
4 & $\mathrm{CH}_{3}$ & $\mathrm{CH}_{3}$ & VI-Ad & 78.6 \\
5 & $\mathrm{H}$ & $\mathrm{H}$ & VI-Ba & 80.7 \\
6 & $\mathrm{H}$ & $\mathrm{CH}_{3}$ & VI-Bb & 82.2 \\
7 & $\mathrm{CH}_{3}$ & $\mathrm{H}$ & VI-Bc & 83.2 \\
8 & $\mathrm{CH}_{3}$ & $\mathrm{CH}_{3}$ & VI-Bd & 81.7 \\
\hline
\end{tabular}

${ }^{a}$ Reaction conditions: V-A/V-B $(1.0 \mathrm{mmol}), \mathrm{Pd}(\mathrm{OAc})_{2}(0.04 \mathrm{mmol}), \mathrm{IPr} \cdot \mathrm{HCl}$ $(0.08 \mathrm{mmol})$ and $\mathrm{K}_{2} \mathrm{CO}_{3}(1.0 \mathrm{mmol})$ were mixed in $3 \mathrm{~mL}$ of NMP at $150{ }^{\circ} \mathrm{C}$ for $8 \mathrm{~h}$ under nitrogen; ${ }^{b}$ Isolated yield.

\section{2 产物 VI 核磁共振的结构鉴定}

从这两系列化合物的 ${ }^{1} \mathrm{H} \mathrm{NMR}\left(\mathrm{CDCl}_{3}\right.$ 为溶剂)结果 来看, 特征峰为 9 号位上亚甲基氢, VI-A 系列的化学位 移在 $\delta 3.81 \sim 3.88$, VI-B 系列在稍高场: $\delta 3.74 \sim 3.79$; 而 ${ }^{13} \mathrm{C} \mathrm{NMR}\left(\mathrm{CDCl}_{3}\right.$ 为溶剂)的结果正相反, VI-A 系列亚甲 基的碳在 $\delta 36.26 \sim 36.50$, VI-B 系列在稍低场: $\delta 36.65 \sim$ 36.92. 为了进一步确定结构, 我们以 VI-Aa 和 VI-Ba 分 别作为这两类二芳胺基芴的例子, 运用 ${ }^{1} \mathrm{H}-{ }^{1} \mathrm{H}$ NOESY 和 ${ }^{1} \mathrm{H}-{ }^{1} \mathrm{H}$ COSY 等二维核磁共振技术对其进行解析，以 鉴别这两类化合物的结构差异, 见图 1 和图 2. 需要说 明的是, 以 $\mathrm{CDCl}_{3}$ 为溶剂测试样品时, VI-Aa 芳环区每 个质子的化学位移均能分辨; 而 VI-Ba 峰型严重重叠, 将 VI-Ba 换用 $\mathrm{C}_{6} \mathrm{D}_{6}$ 为溶剂后测试情况有所好转, 但化 学位移整体向高场移动. 

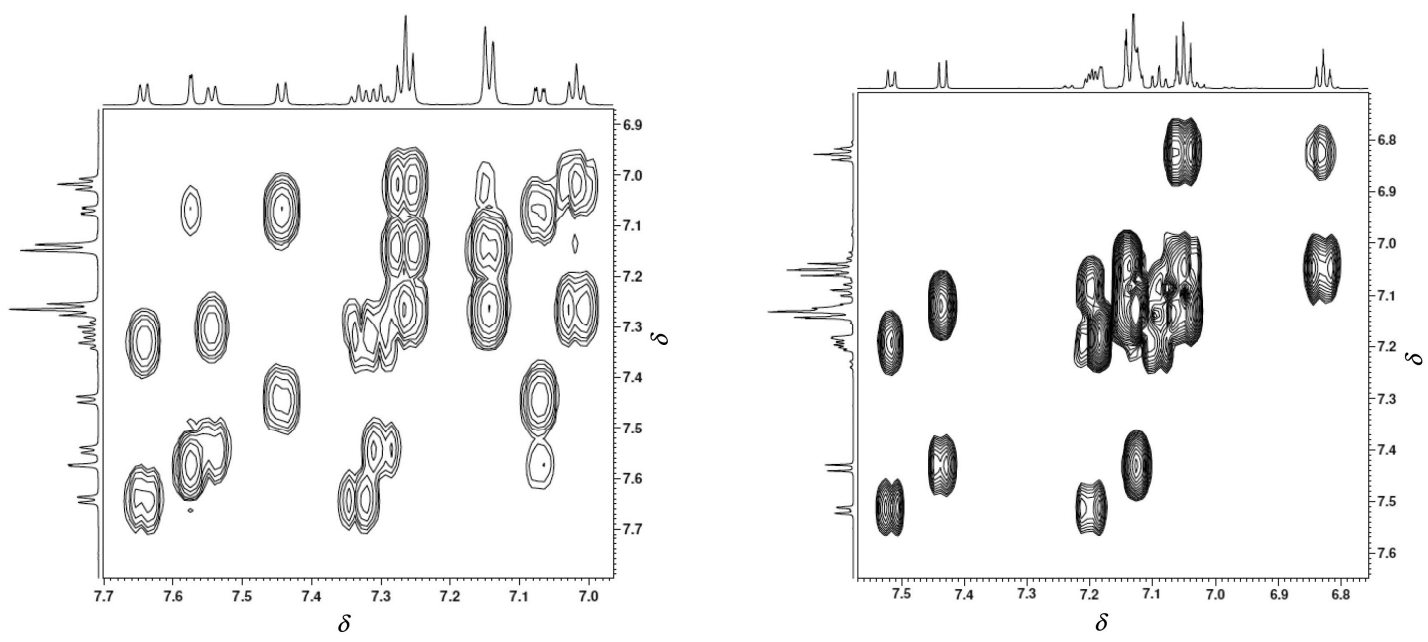

图 1 化合物 VI-Aa 和 VI-Ba 的 ${ }^{1} \mathrm{H}-{ }^{1} \mathrm{H}$ 二维 COSY 谱(低场部分)

Figure $1{ }^{1} \mathrm{H}-{ }^{1} \mathrm{H}$ COSY spectra of VI-Aa and VI-Ba (low field)
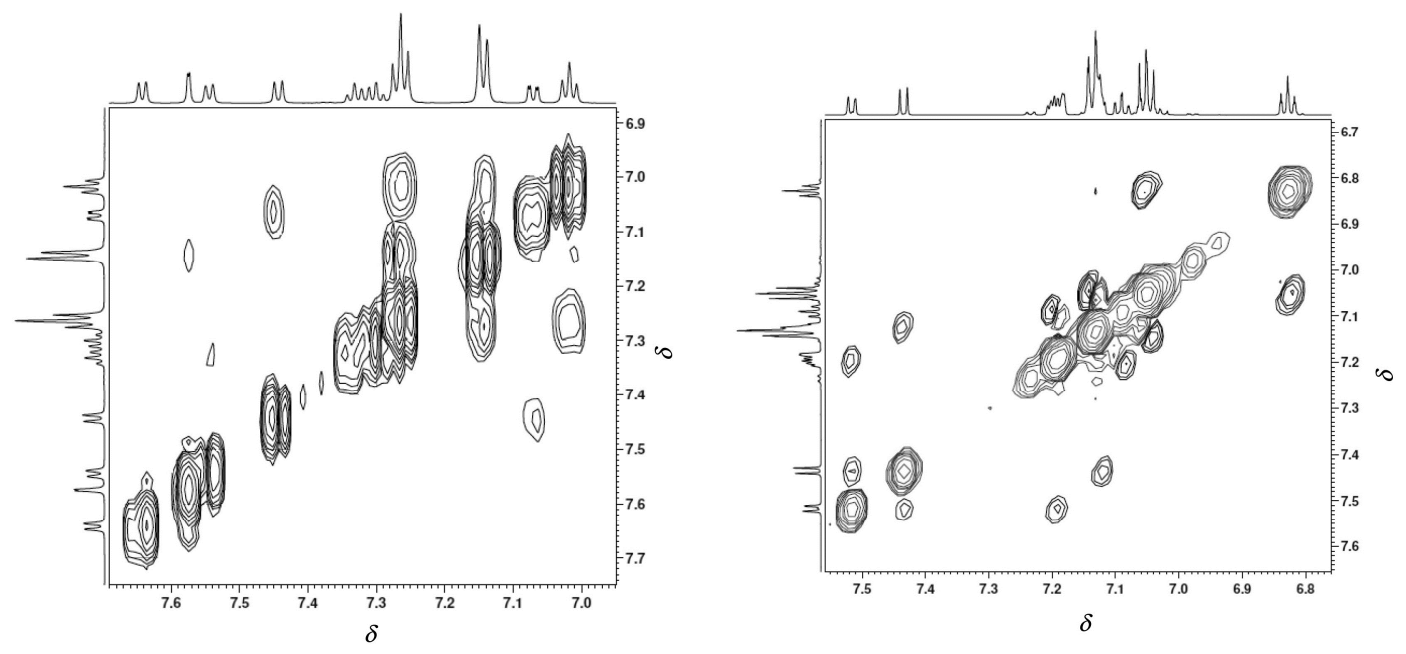

图 2 化合物 VI-Aa 和 VI-Ba 的 ${ }^{1} \mathrm{H}-{ }^{1} \mathrm{H}$ 二维 NOESY 谱(低场部分)

Figure $2{ }^{1} \mathrm{H}-{ }^{1} \mathrm{H}$ NOESY spectra of VI-Aa and VI-Ba (Low field)

对照 VI-Aa 的结构式与 ${ }^{1} \mathrm{H}-{ }^{1} \mathrm{H}$ COSY 谱图, 可以判 断 $\delta 7.55$ 处, $J$ 值为 $1.6 \mathrm{~Hz}$ (间位小裂分) 的 $\mathrm{d}$ 峰为 $\mathrm{H}(4)$ 的 峰. 由此可以确定与之间位相关的 $\mathrm{H}(2)$ 为 $\delta 7.05$ 处的 $\mathrm{dd}$ 峰 $(J=8.0,1.6 \mathrm{~Hz})$, 与 $\mathrm{H}(2)$ 邻位的 $\mathrm{H}(1)$ 为 $\delta 7.44$ 处的 $\mathrm{d}$ 峰. 从 VI-Aa 的 ${ }^{1} \mathrm{H}-{ }^{1} \mathrm{H}$ NOESY 谱图可以发现, 亚甲基 $\mathrm{H}(9)$ 不仅与 $\mathrm{H}(1)$ 相关, 也与 $\mathrm{H}(8)$ 相关; $\mathrm{H}(4)$ 与 $\delta 7.63$ 处 的 $\mathrm{H}(5)$ 相关. 可以清楚地分辨出 VI-Aa 芴环上的质子.

对照 VI-Ba 的结构式与 ${ }^{1} \mathrm{H}-{ }^{1} \mathrm{H}$ COSY 和 ${ }^{1} \mathrm{H}-{ }^{1} \mathrm{H}$ NOESY 谱图可以判断, $\delta 7.18$ 处 $J$ 值为 $1.6 \mathrm{~Hz}$ 的 $\mathrm{d}$ 峰为 $\mathrm{H}(1)$ 的峰, 与之间位相关的 $\mathrm{H}(3)$ 特征峰未找到, 可能包 在了 $\delta 7.16$ 处的溶剂 $\left(\mathrm{C}_{6} \mathrm{D}_{6}\right)$ 残留峰里, 而该处与 $\delta 7.44$ 处的 $\mathrm{H}(4)$ 有相关峰也证实了这一点. $\delta 7.44$ 处的 $\mathrm{H}(4)$ 与 $\delta 7.52$ 在 COSY 中没有相关峰, 但却有 NOESY 的相关 峰, 这说明 $\delta 7.52$ 处是 $\mathrm{H}(5)$ 的峰, 同样亚甲基 $\mathrm{H}(9)$ 不仅 与 $\mathrm{H}(1)$ 相关也与 $\mathrm{H}(8)$ 相关. 通过以上解析, 可以把 VI-Aa 与 VI-Ba 芴环上的各个 H 进行归属(表 3).
表 3 化合物 VI-Aa 与 VI-Ba 的 ${ }^{1} \mathrm{H}$ NMR 核磁共振数据 $(\delta)^{a}$ Table 3 Data of ${ }^{1} \mathrm{H}$ NMR $(\delta)$ of VI-Aa/VI-Ba

\begin{tabular}{|c|c|c|}
\hline No. & $\left(\mathrm{CDCl}_{3}\right)$ & $\left(\mathrm{C}_{6} \mathrm{D}_{6}\right)$ \\
\hline $\mathrm{H}(1)$ & $7.44, \mathrm{~d}$ & $7.18, d$ \\
\hline $\mathrm{H}(2)$ & $7.05, \mathrm{dd}$ & - \\
\hline $\mathrm{H}(3)$ & - & 7.14 左右, 包在溶剂峰里 \\
\hline $\mathrm{H}(4)$ & $7.55, \mathrm{~d}$ & $7.44, \mathrm{~d}$ \\
\hline $\mathrm{H}(5)$ & $7.63, d$ & $7.52, \mathrm{~d}$ \\
\hline $\mathrm{H}(6)$ & $7.32, \mathrm{t}$ & $7.19, \mathrm{t}$ \\
\hline $\mathrm{H}(7)$ & $7.29, \mathrm{t}$ & $7.09, \mathrm{t}$ \\
\hline $\mathrm{H}(8)$ & $7.52, \mathrm{~d}$ & $7.20, \mathrm{~d}$ \\
\hline $\mathrm{H}(9)$ & $3.88, \mathrm{~s}$ & $3.35, \mathrm{~s}$ \\
\hline a & /I-Aa & VI-Ba \\
\hline
\end{tabular}




\section{3 产物 $\mathrm{VI}$ 的红外光谱}

对 VI-A 和 VI-B 系列化合物进行了红外光谱测试. 由表 4 我们可以看到芳环上的 $=\mathrm{C}-\mathrm{H}$ 的伸缩振动频率 在 3016 3034 $\mathrm{cm}^{-1}, \mathrm{C}=\mathrm{C}$ 键的伸缩振动频率在 1585 $1607 \mathrm{~cm}^{-1}, \mathrm{C}-\mathrm{N}$ 键的伸缩振动频率在 $1308 \sim 1319$ $\mathrm{cm}^{-1}$. 无论 VI-A 系列还是 VI-B 系列, 当二苯胺基上的 氢原子被甲基取代时，由于甲基的推电子作用，使得整 个分子共轭体系电子云密度增加, $\mathrm{C}=\mathrm{C}$ 键的伸缩振动 频率向高频方向移动; $\mathrm{C}-\mathrm{N}$ 键的伸缩振动频率也稍向 高频方向移动.

表 4 产物 VI-A/VI-B 的红外光谱

Table 4 IR spectral data of VI-A/VI-B

\begin{tabular}{cccc}
\hline \multirow{2}{*}{ 化合物 } & \multicolumn{3}{c}{$\mathrm{IR} v / \mathrm{cm}^{-1}$} \\
\cline { 2 - 4 } & $=\mathrm{C}-\mathrm{H}$ & $\mathrm{C}=\mathrm{C}$ & $\mathrm{C}-\mathrm{N}$ \\
\hline VI-Aa & 3025 & 1587 & 1308 \\
VI-Ab & 3030 & 1588 & 1310 \\
VI-Ac & 3020 & 1606 & 1313 \\
VI-Ad & 3025 & 1607 & 1317 \\
VI-Ba & 3034 & 1585 & 1313 \\
VI-Bb & 3030 & 1590 & 1310 \\
VI-Bc & 3020 & 1606 & 1319 \\
VI-Bd & 3016 & 1606 & 1316 \\
\hline
\end{tabular}

\section{4 产物 VI 的紫外吸收光谱}

图 3 为 VI-A 与 VI-B 系列化合物在 $\mathrm{CH}_{2} \mathrm{Cl}_{2}$ 溶液中 的紫外-可见吸收光谱, 其最大吸收峰在 301 333 nm. 相对于 VI-A 系列目标产物来说, VI-B 系列目标产物由 于分子的四个苯环结构均处于共轭状态, 增加了分子的 共轭范围, 使得 VI-B 系列的目标化合物紫外吸收波长 红移至 $330 \mathrm{~nm}$ 左右. 此外, 我们可以明显观察 VI-A 系 列目标产物的最大吸收峰在 $305 \mathrm{~nm}$ 左右. 而 VI-B 系列 目标产物的紫外吸收峰明显变宽. 这主要是因为 VI-B 系列目标产物共轭面积大于 VI-A 系列, 其 HOMO 和 LUMO 的轨道重叠更多, 使得 HOMO $\rightarrow$ LUMO 之间的 电子跃迁更为容易，导致峰型变宽.

\section{5 产物 VI 的荧光光谱}

VI-A 和 VI-B 系列化合物的荧光光谱测试显示, 其 苂光发射波长 $\lambda_{\mathrm{em}}$ 在 387 415 $\mathrm{nm}$ (表 5), 并都有一定的 Stokes 位移. 实验所测得的苂光发射光谱与相应的紫外 可见光吸收光谱一一对应.

\section{2 结论}

利用不同金属及配体催化活性和 $\mathrm{I} 、 \mathrm{Br} 、 \mathrm{Cl}$ 三个卤 素原子反应活性的差异, 通过串联的铜、钯金属催化的 三步反应得到了 4 个 2 位取代的二芳胺基茀和 4 个 3 位 取代的二芳胺基荡。这些化合物的结构均通过了 ${ }^{1} \mathrm{H}$ NMR, ${ }^{13} \mathrm{C}$ NMR, HRMS 和 IR 等手段的表征. 对其中

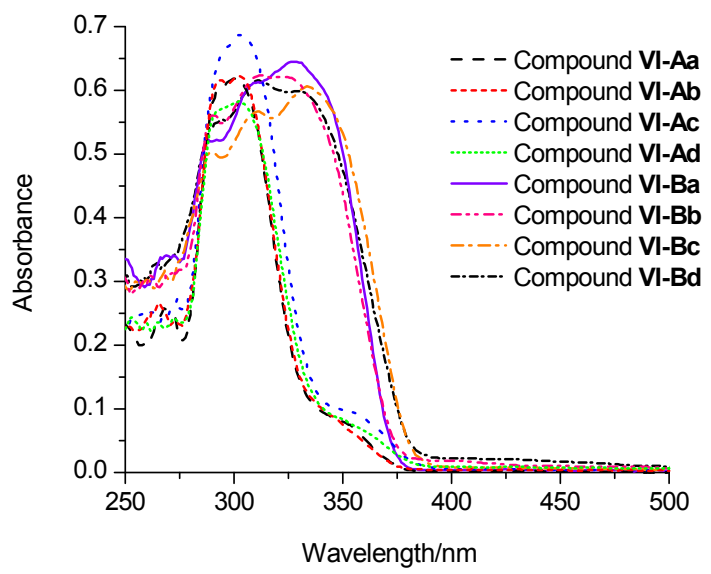

图 3 产物 VI-A 和 VI-B 在二氯甲烷溶液中的紫外吸收光谱 $\left(2.5 \times 10^{-5} \mathrm{~mol} / \mathrm{L}\right.$, r.t. $)$

Figure 3 UV-visble spectra of compound VI-A and VI-B in $\mathrm{CH}_{2} \mathrm{Cl}_{2}\left(2.5 \times 10^{-5} \mathrm{~mol} / \mathrm{L}\right.$, r.t. $)$

表 5 产物 VI-A/VI-B 的苂光光谱

Table 5 Fluorescence properties of VI-A/VI-B

\begin{tabular}{ccccc}
\hline Entry & Compd. & $\lambda_{\text {abs }} / \mathrm{nm}$ & $\lambda_{\text {flu }} / \mathrm{nm}$ & ${\text { Stoke shift } / \mathrm{cm}^{-1}}^{-1}$ \\
\hline 1 & VI-Aa & 301 & 387 & $1.16 \times 10^{5}$ \\
2 & VI-Ab & 306 & 415 & $0.92 \times 10^{5}$ \\
3 & VI-Ac & 305 & 389 & $1.19 \times 10^{5}$ \\
4 & VI-Ad & 303 & 410 & $0.94 \times 10^{5}$ \\
5 & VI-Ba & 327 & 389 & $1.61 \times 10^{5}$ \\
6 & VI-Bb & 323 & 406 & $1.21 \times 10^{5}$ \\
7 & VI-Bc & 332 & 387 & $1.82 \times 10^{5}$ \\
8 & VI-Bd & 313 & 403 & $1.11 \times 10^{5}$ \\
\hline
\end{tabular}

两个具有代表性的二芳胺基芴的结构运用二维核磁共 振技术进行了解析. 然后对这一系列化合物进行了光谱 性能的测试, 发现 VI-B 系列产物较 VI-A 系列产物，具 有更长的紫外吸收波长 $(311 \sim 333 \mathrm{~nm})$. 这些数据可为 进一步开发二芳胺基芴类发光材料作参考.

\section{3 实验部分}

\section{1 仪器与试剂}

熔点仪：上海精科仪器有限公司 SGW X-4 显微熔 点仪，温度计未校正; 核磁共振仪：瑞士 BRUKER 公司 AVANCE III $(400 \mathrm{MHz})$ 型核磁共振仪, 美国 AVANCE 公司 AVANCE DMX $(500 \mathrm{MHz})$ 核磁共振仪, 美国 Vairan 公司 $700 \mathrm{MHz}$ premium shielded 核磁共振仪, $\mathrm{CDCl}_{3}$ 为溶剂, $\mathrm{TMS}$ 为内标; 高分辨质谱仪: Bruker Daltonics micrOTOF II; 红外光谱仪: Nicolet 6700 型; 紫外光谱仪: Shimadzu UV-2450; 荧光光谱仪: Cary Eclipse (varian), 激光器: 天津市东港科技发展有限公 司 GY-10. 所有试剂均为分析纯, 使用之前未经进一步 纯化. 


\section{2 实验方法}

\subsubsection{III-A 和 III-B 系列化合物的合成}

以 3-溴-4-甲基三苯胺(III-Aa $)^{[30]}$ 为例：在一个 50 $\mathrm{mL}$ Schlenk 瓶中, 在氮气保护下, 依次加入 3-溴-4-甲基 苯胺 $558 \mathrm{mg}(3 \mathrm{mmol})$ 、碘苯 $1.53 \mathrm{~g}(7.5 \mathrm{mmol})$ 、甲苯 5 $\mathrm{ml} 、 1,10$ - 菲啰啉 $119 \mathrm{mg}(0.6 \mathrm{mmol}) 、$ 氯化亚铜 $59 \mathrm{mg}(0.6$ $\mathrm{mmol})$ 、氢氧化钾 $1.4 \mathrm{~g}(24 \mathrm{mmol})$. 搅拌回流反应 $24 \mathrm{~h}$, 冷却反应液, 回收甲苯, 硅胶柱层析分离得到 $827 \mathrm{mg}$ 白 色固体 III-Aa, 产率 81.8\%. m.p. 112 $114{ }^{\circ} \mathrm{C} ;{ }^{1} \mathrm{H}$ NMR $\left(\mathrm{CDCl}_{3}, 400 \mathrm{MHz}\right) \delta: 2.35(\mathrm{~s}, 3 \mathrm{H}), 6.93(\mathrm{dd}, J=8.0,2.0$ $\mathrm{Hz}, 1 \mathrm{H}), 7.00 \sim 7.10(\mathrm{~m}, 7 \mathrm{H}), 7.22 \sim 7.27(\mathrm{~m}, 5 \mathrm{H}) ;{ }^{13} \mathrm{C}$ NMR (125 MHz, $\left.\mathrm{CDCl}_{3}\right) \delta: 22.98,117.71,123.00,124.28$, $126.06,129.32,132.85,138.66,147.16,147.55$.

4,4'-二甲基-3"-溴-4"-甲基三苯胺(III-Ab) ${ }^{[31]}$ : 白色 固体, 产率 81.1\%. m.p. $115 \sim 118{ }^{\circ} \mathrm{C} ;{ }^{1} \mathrm{H}$ NMR $\left(\mathrm{CDCl}_{3}\right.$, $400 \mathrm{MHz}) \delta: 2.31(\mathrm{~s}, 6 \mathrm{H}), 2.33(\mathrm{~s}, 3 \mathrm{H}), 6.86(\mathrm{dd}, J=8.0$, $2.2 \mathrm{~Hz}, 1 \mathrm{H}), 6.96(\mathrm{~d}, J=8.2 \mathrm{~Hz}, 4 \mathrm{H}), 7.02 \sim 7.05(\mathrm{~m}, 1 \mathrm{H})$, $7.06(\mathrm{~d}, J=8.2 \mathrm{~Hz}, 4 \mathrm{H}), 7.21(\mathrm{~d}, J=2.2 \mathrm{~Hz}, 1 \mathrm{H}) ;{ }^{13} \mathrm{C}$ NMR (125 MHz, $\left.\mathrm{CDCl}_{3}\right) \delta: 20.80,22.96,116.51,121.76$, $124.47,124.76,129.88,132.55,132.59,138.31,145.02$, 147.46 .

3- 甲基-4-溴三苯胺(III-Ba $)^{[32]}$ : 白色固体, 产率 83.7\%. m.p. $135 \sim 138{ }^{\circ} \mathrm{C} ;{ }^{1} \mathrm{H} \mathrm{NMR}\left(\mathrm{CDCl}_{3}, 400 \mathrm{MHz}\right) \delta$ : $2.28(\mathrm{~s}, 3 \mathrm{H}), 6.76(\mathrm{dd}, J=8.6,2.6 \mathrm{~Hz}, 1 \mathrm{H}), 6.95(\mathrm{~d}, J=2.6$ $\mathrm{Hz}, 1 \mathrm{H}), 7.00 \sim 7.07(\mathrm{~m}, 6 \mathrm{H}), 7.22 \sim 7.27(\mathrm{~m}, 4 \mathrm{H}), 7.35(\mathrm{~d}$, $J=8.6 \mathrm{~Hz}, 1 \mathrm{H}) ;{ }^{13} \mathrm{C} \mathrm{NMR}\left(125 \mathrm{MHz}, \mathrm{CDCl}_{3}\right) \delta: 22.12$, $122.98,123.01,124.14,125.04,127.52,129.31,131.02$, $131.90,146.83,147.47$.

4,4'-二甲基-3"-甲基-4"-溴三苯胺(III-Bb $)^{[33]}$ : 白色 固体, 产率 80.3\%. m.p. $128 \sim 131{ }^{\circ} \mathrm{C} ;{ }^{1} \mathrm{H}$ NMR $\left(\mathrm{CDCl}_{3}\right.$, $400 \mathrm{MHz}) \delta: 2.27(\mathrm{~s}, 3 \mathrm{H}), 2.31(\mathrm{~s}, 6 \mathrm{H}), 6.71(\mathrm{dd}, J=8.6$, $2.8 \mathrm{~Hz}, 1 \mathrm{H}), 6.91(\mathrm{~d}, J=2.8 \mathrm{~Hz}, 1 \mathrm{H}), 6.96(\mathrm{~d}, J=8.0 \mathrm{~Hz}$, 4H), $7.06(\mathrm{~d}, J=8.0 \mathrm{~Hz}, 4 \mathrm{H}), 7.31(\mathrm{~d}, J=8.6 \mathrm{~Hz}, 1 \mathrm{H}) ;{ }^{13} \mathrm{C}$ NMR (125 MHz, $\left.\mathrm{CDCl}_{3}\right) \delta: 20.81,22.06,121.87,124.43$, $125.00,126.30,129.96,130.82,130.87,132.67,145.08$, 147.32 .

\section{2 .2 化合物 V-A 和 V-B 的合成}

以 3-(2-氯苯基)-4-甲基三苯胺( $\mathbf{V}-\mathbf{A a})$ 为例：在一个 $25 \mathrm{~mL}$ Schlenk 瓶中, 在氮气保护下, 依次加入异丙醇 5 $\mathrm{mL}$ 、二氯二(三苯基膦)钯 $14 \mathrm{mg}(0.02 \mathrm{mmol})$ 、碳酸钾 $553 \mathrm{mg}$ (4 mmol), 室温下搅拌 $5 \mathrm{~min}$. 再加入邻氯苯嗍 酸 $375 \mathrm{mg}$ (2.4 mmol)、 $N, N$-二苯基-4-甲基-3-溴苯胺 676 $\mathrm{mg}(2 \mathrm{mmol})$, 反应物加热回流 $6 \mathrm{~h}$ 后停止. 冷却反应液, 水泵减压除去溶剂. 将残余物拌硅胶, 硅胶柱层析分离, 得到 $694 \mathrm{mg}$ 白色粉末状固体 V-Aa, 产率 94.1\%. m.p.
133 $136{ }^{\circ} \mathrm{C}$; UV-vis $\left(\mathrm{CH}_{2} \mathrm{Cl}_{2}\right) \lambda_{\max }: 300 \mathrm{~nm}$; ${ }^{1} \mathrm{H}$ NMR $\left(\mathrm{CDCl}_{3}, 400 \mathrm{MHz}\right) \delta: 2.09(\mathrm{~s}, 3 \mathrm{H}), 6.94 \sim 7.05(\mathrm{~m}, 4 \mathrm{H})$, $7.11 \sim 7.18(\mathrm{~m}, 5 \mathrm{H}), 7.21 \sim 7.24(\mathrm{~m}, 7 \mathrm{H}), 7.42 \sim 7.44(\mathrm{~m}$, $1 \mathrm{H}) ;{ }^{13} \mathrm{C} \mathrm{NMR}\left(125 \mathrm{MHz}, \mathrm{CDCl}_{3}\right) \delta: 19.10,122.39$, $123.83,124.04,125.73,126.46,128.51,129.12,129.39$, $130.63,130.76,130.93,133.37,140.18,140.27,145.26$, 147.89; IR (KBr) v: 3022, 2921, 1585, 1491, 1471, 1333, 1266, 751, 696; HRMS calcd for $\mathrm{C}_{25} \mathrm{H}_{20}{ }^{35} \mathrm{ClN}\left(\mathrm{M}^{+}+\mathrm{H}\right)$ 370.1363 , found 370.1365 .

3-(2-氯-4-甲基苯基)-4-甲基三苯胺( $\mathbf{V}-\mathbf{A b})$ : 白色粉 末状固体，产率 96.5\%. m.p. $127 \sim 130{ }^{\circ} \mathrm{C}$; UV-vis $\left(\mathrm{CH}_{2} \mathrm{Cl}_{2}\right) \lambda_{\max }: 300 \mathrm{~nm} ;{ }^{1} \mathrm{H} \mathrm{NMR}\left(\mathrm{CDCl}_{3}, 400 \mathrm{MHz}\right) \delta$ : $2.08(\mathrm{~s}, 3 \mathrm{H}), 2.35$ (s, 3H), $6.91(\mathrm{~d}, J=2.4 \mathrm{~Hz}, 1 \mathrm{H}), 6.94 \sim$ $7.02(\mathrm{~m}, 3 \mathrm{H}), 7.05 \sim 7.16(\mathrm{~m}, 7 \mathrm{H}), 7.20 \sim 7.23(\mathrm{~m}, 5 \mathrm{H})$; $\left.{ }^{13} \mathrm{C} \mathrm{NMR} \mathrm{(125} \mathrm{MHz,} \mathrm{CDCl}_{3}\right) \delta: 19.15,20.83,122.30$, $123.75,123.80,125.85,127.25,129.07,129.75,130.51$, $130.58,130.85,132.89,137.14,138.60,140.08,145.11$, 147.80; IR (KBr) v: 3028, 2918, 1585, 1487, 1333, 1269, 1070, 822, 753, 694; HRMS calcd for $\mathrm{C}_{26} \mathrm{H}_{22}{ }^{35} \mathrm{CIN}$ 383.1441 , found 383.1444 .

4,4'-二甲基-3"-(2-氯苯基)-4"-甲基三苯胺( V-Ac): 白色粉末状固体，产率 92.7\%. m.p. 139 142 ${ }^{\circ} \mathrm{C}$; UV-vis $\left(\mathrm{CH}_{2} \mathrm{Cl}_{2}\right) \lambda_{\max }: 302 \mathrm{~nm} ;{ }^{1} \mathrm{H}$ NMR $\left(\mathrm{CDCl}_{3}, 400\right.$ $\mathrm{MHz}) \delta: 2.08(\mathrm{~s}, 3 \mathrm{H}), 2.31(\mathrm{~s}, 6 \mathrm{H}), 6.90 \sim 6.92(\mathrm{~m}, 1 \mathrm{H})$, $6.97 \sim 6.07(\mathrm{~m}, 9 \mathrm{H}), 7.12 \sim 7.15(\mathrm{~m}, 1 \mathrm{H}), 7.21 \sim 7.28(\mathrm{~m}$, $3 \mathrm{H}), 7.41 \sim 7.44(\mathrm{~m}, 1 \mathrm{H}) ;{ }^{13} \mathrm{C} \mathrm{NMR}\left(125 \mathrm{MHz}, \mathrm{CDCl}_{3}\right) \delta$ : $19.01,20.73,122.89,124.11,124.53,126.42,128.42$, $129.34,129.76,129.93,130.42,130.97,131.95,133.40$, 139.98, 140.46, 145.53, 145.69; IR (KBr) v: 3023, 2915, 1604, 1506, 1470, 1324, 1276, 1032, 813, 744; HRMS calcd for $\mathrm{C}_{27} \mathrm{H}_{24}{ }^{35} \mathrm{ClN} \quad\left(\mathrm{M}^{+}+\mathrm{H}\right)$ 398.1676, found 398.1659.

4,4'-二甲基-3"-(2-氯-4-甲基苯基)-4"-甲基三苯胺 (V-Ad): 白色粉末状固体，产率 96.1\%. m.p. 137 $140{ }^{\circ} \mathrm{C}$; UV-vis $\left(\mathrm{CH}_{2} \mathrm{Cl}_{2}\right) \lambda_{\text {max }}: 300 \mathrm{~nm} ;{ }^{1} \mathrm{H} \mathrm{NMR}\left(\mathrm{CDCl}_{3}\right.$, $400 \mathrm{MHz}) \delta: 2.05(\mathrm{~s}, 3 \mathrm{H}), 2.28(\mathrm{~s}, 6 \mathrm{H}), 2.34(\mathrm{~s}, 3 \mathrm{H}), 6.85$ $(\mathrm{d}, J=2.0 \mathrm{~Hz}, 1 \mathrm{H}), 6.95$ (dd, $J=8.4,2.2 \mathrm{~Hz}, 1 \mathrm{H}), 6.98 \sim$ $7.04(\mathrm{~m}, 7 \mathrm{H}), 7.05 \sim 7.11(\mathrm{~m}, 4 \mathrm{H}), 7.23(\mathrm{~s}, 1 \mathrm{H}) ;{ }^{13} \mathrm{C} \mathrm{NMR}$ $\left(125 \mathrm{MHz}, \mathrm{CDCl}_{3}\right) \delta: 19.08,20.75,20.84,122.63,124.05$, $124.62,127.22,129.71,129.78,130.30,130.62,131.86$, $132.92,137.34,138.49,139.88,145.43,145.56$; IR (KBr) $v: 3022,2919,1603,1505,1481,1324,1270,818$; HRMS calcd for $\mathrm{C}_{28} \mathrm{H}_{26}{ }^{35} \mathrm{ClN} 411.1754$, found 411.1755.

3-甲基-4-(2-氯苯基)三苯胺(V-Ba): 白色粉末状固 体, 产率 96.5\%. m.p. $137 \sim 139{ }^{\circ} \mathrm{C}$; UV-vis $\left(\mathrm{CH}_{2} \mathrm{Cl}_{2}\right)$ 
$\lambda_{\text {max }}: 302 \mathrm{~nm} ;{ }^{1} \mathrm{H}$ NMR (CDCl3, $\left.400 \mathrm{MHz}\right) \delta: 2.03$ (s, 3H), $6.92 \sim 6.95(\mathrm{~m}, 1 \mathrm{H}), 6.98 \sim 7.05(\mathrm{~m}, 4 \mathrm{H}), 7.15(\mathrm{~d}, J=8.4$ $\mathrm{Hz}, 4 \mathrm{H}), 7.25 \sim 7.31(\mathrm{~m}, 7 \mathrm{H}), 7.45 \sim 7.48(\mathrm{~m}, 1 \mathrm{H}) ;{ }^{13} \mathrm{C}$ NMR (125 MHz, $\left.\mathrm{CDCl}_{3}\right) \delta: 19.92,120.66,122.79,124.50$, $126.53,126.51,128.38,129.22,129.37,130.22,131.43$, 133.68, 133.77, 137.18, 140.48, 147.27, 147.83; IR (KBr) $v: 3032,2920,1590,1493,1465,1332,1270,752,694$; HRMS calcd for $\mathrm{C}_{25} \mathrm{H}_{20}{ }^{35} \mathrm{ClN}\left(\mathrm{M}^{+}+\mathrm{H}\right) 370.1363$, found 370.1351 .

3-甲基-4-(2-氯-4-甲基苯基)三苯胺( $\mathbf{V}$-Bb): 白色粉 末状固体, 产率 92.6\%. m.p. $142 \sim 146{ }^{\circ} \mathrm{C}$; UV-vis $\left(\mathrm{CH}_{2} \mathrm{Cl}_{2}\right) \lambda_{\max }: 300 \mathrm{~nm} ;{ }^{1} \mathrm{H}$ NMR $\left(\mathrm{CDCl}_{3}, 400 \mathrm{MHz}\right) \delta$ : $2.02(\mathrm{~s}, 3 \mathrm{H}), 2.38(\mathrm{~s}, 3 \mathrm{H}), 6.90 \sim 7.02(\mathrm{~m}, 5 \mathrm{H}), 7.05 \sim 7.16$ $(\mathrm{m}, 6 \mathrm{H}), 7.25 \sim 7.28(\mathrm{~m}, 5 \mathrm{H}) ;{ }^{13} \mathrm{C}$ NMR $(125 \mathrm{MHz}$, $\left.\mathrm{CDCl}_{3}\right) \delta: 19.95,20.86,120.67,122.70,124.41,124.53$, 127.34, 129.19, 129.79, 130.38, 131.10, 133.32, 133.63, 137.31, 137.36, 138.47, 147.08, 147.80; IR (KBr) v: 3026, 2920, 1590, 1491, 1481, 1318, 1270, 1068, 813, 753, 696; HRMS calcd for $\mathrm{C}_{26} \mathrm{H}_{22}{ }^{35} \mathrm{ClN} 383.1441$, found 383.1444.

4,4'-二甲基-3"-甲基-4"-(2-氯苯基)三苯胺( V-Bc): 白色粉末状固体, 产率 94.4\%. m.p. $136 \sim 138{ }^{\circ} \mathrm{C}$; UV-vis $\left(\mathrm{CH}_{2} \mathrm{Cl}_{2}\right) \lambda_{\max }: 300 \mathrm{~nm} ;{ }^{1} \mathrm{H}$ NMR $\left(\mathrm{CDCl}_{3}, 400\right.$ MHz) $\delta: 2.00(\mathrm{~s}, 3 \mathrm{H}), 2.32(\mathrm{~s}, 6 \mathrm{H}), 6.86(\mathrm{~d}, J=8.4 \mathrm{~Hz}$, $1 \mathrm{H}), 6.92 \sim 6.97(\mathrm{~m}, 2 \mathrm{H}), 7.03 \sim 7.09(\mathrm{~m}, 8 \mathrm{H}), 7.25 \sim 7.30$ $(\mathrm{m}, 3 \mathrm{H}), 7.43 \sim 7.46(\mathrm{~m}, 1 \mathrm{H}) ;{ }^{13} \mathrm{C}$ NMR $(125 \mathrm{MHz}$, $\left.\mathrm{CDCl}_{3}\right) \delta: 19.94,20.82,119.28,123.08,124.79,126.46$, $128.27,129.31,129.85,130.03,131.48,132.41,132.59$, 133.78, 136.91, 140.56, 145.31, 147.63; IR (KBr) v: 3022, 2915, 1603, 1506, 1467, 1324, 1269, 813, 753; HRMS calcd for $\mathrm{C}_{27} \mathrm{H}_{24}{ }^{35} \mathrm{ClN}$ 397.1597, found 397.1595.

4,4'-二甲基-3"- 甲基-4"-(2-氯-4-甲基苯基)三苯胺 (V-Bd): 白色粉末状固体, 产率 93.7\%. m.p. 161 $164{ }^{\circ} \mathrm{C}$; UV-vis $\left(\mathrm{CH}_{2} \mathrm{Cl}_{2}\right) \lambda_{\text {max }}: 304 \mathrm{~nm} ;{ }^{1} \mathrm{H} \mathrm{NMR}\left(\mathrm{CDCl}_{3}\right.$, $400 \mathrm{MHz}) \delta: 2.00$ (s, 3H), 2.32 (s, 6H), 2.37 (s, 3H), 6.85 $(\mathrm{d}, J=8.0 \mathrm{~Hz}, 1 \mathrm{H}), 6.91 \sim 6.95(\mathrm{~m}, 2 \mathrm{H}), 7.02 \sim 7.09(\mathrm{~m}$, 9H), $7.12(\mathrm{t}, J=7.6 \mathrm{~Hz}, 1 \mathrm{H}), 7.27(\mathrm{~s}, 1 \mathrm{H}) ;{ }^{13} \mathrm{C}$ NMR $(125$ $\left.\mathrm{MHz}, \mathrm{CDCl}_{3}\right) \delta: 19.99,20.83,20.87,119.30,123.10$, $124.73,127.31,129.74,129.83,130.02,131.18,132.35$, 133.36, 137.06, 137.45, 138.35, 145.30, 147.45; IR (KBr) v: 3022, 2919, 1603, 1505, 1481, 1324, 1270, 818; HRMS calcd for $\mathrm{C}_{28} \mathrm{H}_{26}{ }^{35} \mathrm{ClN} 411.1754$, found 411.1757 .

\subsection{3 化合物 VI-A 和 VI-B 的合成}

以 3-二苯胺基芴(VI-Aa)的制备为例: 在一个 $25 \mathrm{~mL}$ Schlenk 瓶中, 在氮气保护下, 依次加入中间产物 V-Aa $554 \mathrm{mg}(1.5 \mathrm{mmol})$ 、醋酸钯 $13.5 \mathrm{mg}(0.06 \mathrm{mmol}) 、 1,3-$
双 $(2,6$-二异丙基苯基)咪唑鎓氯盐 $50.9 \mathrm{mg}(0.12 \mathrm{mmol})$ 、 碳酸钾 $207 \mathrm{mg}(1.5 \mathrm{mmol})$ 和 $N$-甲基吡咯烷酮 $3 \mathrm{~mL}$. 反 应物在 $150{ }^{\circ} \mathrm{C}$ 下反应 $8 \mathrm{~h}$ 后停止. 冷却反应液, 向反应 体系中加入 $10 \mathrm{~mL}$ 水, 并用乙酸乙酯 $(5 \mathrm{~mL} \times 3)$ 萃取, 合 并有机相, 无水硫酸镁干燥后, 回收溶剂. 用硅胶柱层 析分离, 得到 $417 \mathrm{mg}$ 亮黄色固体 VI-Aa, 产率 83.4\%. m.p. $130 \sim 134{ }^{\circ} \mathrm{C}$; UV-vis $\left(\mathrm{CH}_{2} \mathrm{Cl}_{2}\right) \lambda_{\max }: 301 \mathrm{~nm} ;{ }^{1} \mathrm{H}$ NMR $\left(\mathrm{CDCl}_{3}, 400 \mathrm{MHz}\right) \delta: 3.88(\mathrm{~s}, 2 \mathrm{H}), 7.00$ (t, $J=7.2$ $\mathrm{Hz}, 2 \mathrm{H}), 7.05$ (dd, $J=8.0,1.6 \mathrm{~Hz}, 1 \mathrm{H}), 7.12(\mathrm{t}, J=8.0 \mathrm{~Hz}$, $4 \mathrm{H}), 7.24$ (d, $J=7.2 \mathrm{~Hz}, 4 \mathrm{H}), 7.29$ (t, $J=7.2 \mathrm{~Hz}, 1 \mathrm{H}), 7.32$ (t, $J=7.2 \mathrm{~Hz}, 1 \mathrm{H}), 7.44(\mathrm{~d}, J=8.0 \mathrm{~Hz}, 1 \mathrm{H}), 7.52(\mathrm{~d}, J=7.2$ $\mathrm{Hz}, 1 \mathrm{H}), 7.55(\mathrm{~d}, J=1.6 \mathrm{~Hz}, 1 \mathrm{H}), 7.63(\mathrm{~d}, J=7.2 \mathrm{~Hz}, 1 \mathrm{H})$; ${ }^{13} \mathrm{C}$ NMR $\left(125 \mathrm{MHz}, \mathrm{CDCl}_{3}\right) \delta: 36.50,116.74,120.04$, $122.32,123.70,124.23,125.01,125.69,126.66,126.82$, 129.18, 138.24, 141.42, 143.03, 143.87, 146.98, 148.20; IR (KBr) v: 3025, 2920, 1587, 1489, 1446, 1308, 1280, 751, 694; HRMS calcd for $\mathrm{C}_{25} \mathrm{H}_{19} \mathrm{~N} 333.1517$ found 333.1518 .

3-二苯胺基-7-甲基芴(VI-Ab): 亮黄色固体, 产率 $84.5 \%$. m.p. $138 \sim 142{ }^{\circ} \mathrm{C}$; UV-vis $\left(\mathrm{CH}_{2} \mathrm{Cl}_{2}\right) \lambda_{\text {max }}: 306 \mathrm{~nm}$; ${ }^{1} \mathrm{H}$ NMR $\left(\mathrm{CDCl}_{3}, 400 \mathrm{MHz}\right) \delta: 2.42(\mathrm{~s}, 3 \mathrm{H}), 3.83(\mathrm{~s}, 2 \mathrm{H})$, $6.97 \sim 7.03(\mathrm{~m}, 4 \mathrm{H}), 7.11 \sim 7.13(\mathrm{~m}, 4 \mathrm{H}), 7.25(\mathrm{t}, J=8.0$ $\mathrm{Hz}, 4 \mathrm{H}), 7.35$ (s, 1H), $7.40(\mathrm{~d}, J=8.0 \mathrm{~Hz}, 1 \mathrm{H}), 7.50 \sim 7.53$ $(\mathrm{m}, 2 \mathrm{H}) ;{ }^{13} \mathrm{C}$ NMR $\left(125 \mathrm{MHz}, \mathrm{CDCl}_{3}\right) \delta: 21.60,36.30$, $116.57,119.72,122.20,123.61,123.75,125.59,125.70$, $127.48,129.13,136.65,138.10,138.73,143.07,144.09$, 146.82, 148.17; IR (KBr) v: 3030, 2916, 1588, 1487, 1310, 1273, 812, 752, 696; HRMS calcd for $\mathrm{C}_{26} \mathrm{H}_{21} \mathrm{~N} 347.1674$, found 347.1673 .

3-二(4-甲基苯)胺基芴(VI-Ac)：黄色固体, 产率 82.9\%. m.p. $159 \sim 161{ }^{\circ} \mathrm{C}$; UV-vis $\left(\mathrm{CH}_{2} \mathrm{Cl}_{2}\right) \lambda_{\text {max }}: 305 \mathrm{~nm}$; ${ }^{1} \mathrm{H} \mathrm{NMR}\left(\mathrm{CDCl}_{3}, 400 \mathrm{MHz}\right) \delta: 2.32(\mathrm{~s}, 6 \mathrm{H}), 3.85(\mathrm{~s}, 2 \mathrm{H})$, 6.98 7.07 (m, 9H), 7.26 7.31 (m, 2H), $7.38(\mathrm{~d}, J=8.0$ $\mathrm{Hz}, 1 \mathrm{H}), 7.50 \sim 7.53(\mathrm{~m}, 2 \mathrm{H}), 7.62(\mathrm{~d}, J=8.0 \mathrm{~Hz}, 1 \mathrm{H}) ;{ }^{13} \mathrm{C}$ NMR (125 MHz, $\left.\mathrm{CDCl}_{3}\right) \delta: 20.76,36.41,115.63,119.99$, 123.24, 123.90, 124.97, 125.48, 126.58, 126.68, 129.78, 131.84, 137.31, 141.50, 142.78, 143.90, 145.82, 147.38; IR (KBr) $v: 3020,2918,1606,1507,1484,1448,1313,1273$, 815; HRMS calcd for $\mathrm{C}_{27} \mathrm{H}_{23} \mathrm{~N} 361.1830$, found 361.1831.

3-二(4-甲基苯)胺基-7-甲基芴(VI-Ad): 亮黄色固 体, 产率 78.6\%. m.p. $139 \sim 141{ }^{\circ} \mathrm{C}$; UV-vis $\left(\mathrm{CH}_{2} \mathrm{Cl}_{2}\right)$ $\lambda_{\max }: 303 \mathrm{~nm} ;{ }^{1} \mathrm{H} \mathrm{NMR}\left(\mathrm{CDCl}_{3}, 400 \mathrm{MHz}\right) \delta: 2.32(\mathrm{~s}, 6 \mathrm{H})$, $2.41(\mathrm{~s}, 3 \mathrm{H}), 3.81(\mathrm{~s}, 2 \mathrm{H}), 7.00 \sim 7.07(\mathrm{~m}, 8 \mathrm{H}), 7.17(\mathrm{~s}$, 2H), $7.33 \sim 7.37$ (m, 2H), $7.46(\mathrm{~d}, J=2.4 \mathrm{~Hz}, 1 \mathrm{H}), 7.50$ (d, $J=7.6 \mathrm{~Hz}, 1 \mathrm{H}) ;{ }^{13} \mathrm{C}$ NMR $\left(125 \mathrm{MHz}, \mathrm{CDCl}_{3}\right) \delta: 20.76$, $21.60,36.26,115.41,119.69,123.87,124.05,125.39$, 
$125.68,127.42,129.75,131.74,136.52,137.20,138.88$, 142.87, 144.15, 145.86, 147.29; IR (KBr) v: 3025, 2919, $1607,1507,1485,1317,1268,812$; HRMS calcd for $\mathrm{C}_{28} \mathrm{H}_{25} \mathrm{~N}$ 375.1987, found 375.1988.

2-二苯胺基芴(VI-Ba ${ }^{[34]}$ : 淡黄色固体，产率 $80.7 \%$. m.p. $150 \sim 154{ }^{\circ} \mathrm{C} ;{ }^{1} \mathrm{H}$ NMR $\left(\mathrm{CDCl}_{3}, 400 \mathrm{MHz}\right) \delta: 3.77$ (s, $2 \mathrm{H}), 6.95 \sim 7.15(\mathrm{~m}, 8 \mathrm{H}), 7.29 \sim 7.53(\mathrm{~m}, 7 \mathrm{H}), 7.62 \sim 7.69$ $(\mathrm{m}, 2 \mathrm{H}) ;{ }^{1} \mathrm{H}$ NMR $\left(\mathrm{C}_{6} \mathrm{D}_{6}, 700 \mathrm{~Hz}\right) \delta: 3.35(\mathrm{~s}, 2 \mathrm{H}), 6.83(\mathrm{t}$, $J=7.0 \mathrm{~Hz}, 2 \mathrm{H}), 7.05$ (t, $J=7.0 \mathrm{~Hz}, 4 \mathrm{H}), 7.09$ (t, $J=7.0$ $\mathrm{Hz}, 1 \mathrm{H}), 7.14$ (t, J=7.0 Hz, 4H), $7.13 \sim 7.16(\mathrm{~m}, 1 \mathrm{H}), 7.18$ (d, $J=1.6 \mathrm{~Hz}, 1 \mathrm{H}), 7.19$ (t, $J=7.0 \mathrm{~Hz}, 1 \mathrm{H}), 7.20$ (d, $J=7.0$ $\mathrm{Hz}, 1 \mathrm{H}), 7.44$ (d, $J=7.7 \mathrm{~Hz}, 1 \mathrm{H}), 7.52$ (d, $J=7.7 \mathrm{~Hz}, 1 \mathrm{H})$; ${ }^{13} \mathrm{C}$ NMR (125 MHz, $\left.\mathrm{CDCl}_{3}\right) \delta: 36.92,119.38,120.46$, $121.31,122.59,123.58,124.09,124.33,124.93,126.02$, $126.80,129.25,136.92,141.54,143.14,144.67,146.98$, 148.12 .

2-二苯胺基-7-甲基芴(VI-Bb)：淡黄色固体，产率 82.2\%. m.p. $144 \sim 147{ }^{\circ} \mathrm{C}$; UV-vis $\left(\mathrm{CH}_{2} \mathrm{Cl}_{2}\right) \lambda_{\text {max }}: 323 \mathrm{~nm}$; ${ }^{1} \mathrm{H}$ NMR $\left(\mathrm{CDCl}_{3}, 400 \mathrm{MHz}\right) \delta: 2.42(\mathrm{~s}, 3 \mathrm{H}), 3.77(\mathrm{~s}, 2 \mathrm{H})$, 7.00 (t, $J=7.2 \mathrm{~Hz}, 2 \mathrm{H}), 7.11$ (d, $J=7.6 \mathrm{~Hz}, 5 \mathrm{H}), 7.16$ (d, $J=7.6 \mathrm{~Hz}, 1 \mathrm{H}), 7.25$ (t, $J=8.0 \mathrm{~Hz}, 5 \mathrm{H}), 7.32$ (s, 1H), 7.60 $(\mathrm{t}, J=8.0 \mathrm{~Hz}, 2 \mathrm{H}) ;{ }^{13} \mathrm{C} \mathrm{NMR}\left(125 \mathrm{MHz}, \mathrm{CDCl}_{3}\right) \delta: 21.56$, $36.67,119.04,120.05,121.42,122.37,123.61,123.87$, $125.63,127.55,129.15,135.77,137.05,138.81,143.35$, 144.42, 146.41, 148.08; IR (KBr) v: 3030, 2919, 1590, 1492, 1471, 1310, 1278, 809, 748, 694; HRMS calcd for $\mathrm{C}_{26} \mathrm{H}_{21} \mathrm{~N} 347.1674$, found 347.1676.

2-二(4-甲基苯基)胺基芴(VI-Bc) ${ }^{[35]}$ : 淡黄色固体, 产率 83.2\%. m.p. $122 \sim 126{ }^{\circ} \mathrm{C} ;{ }^{1} \mathrm{H}$ NMR $\left(\mathrm{CDCl}_{3}, 400\right.$ $\mathrm{MHz}) \delta: 2.33(\mathrm{~s}, 6 \mathrm{H}), 3.79(\mathrm{~s}, 2 \mathrm{H}), 7.00 \sim 7.09(\mathrm{~m}, 10 \mathrm{H})$, $7.21 \sim 7.25(\mathrm{~m}, 1 \mathrm{H}), 7.34(\mathrm{t}, J=8.0 \mathrm{~Hz}, 1 \mathrm{H}), 7.48(\mathrm{~d}, J=$ $7.2 \mathrm{~Hz}, 1 \mathrm{H}), 7.61$ (d, $J=8.0 \mathrm{~Hz}, 1 \mathrm{H}), 7.68$ (d, $J=7.6 \mathrm{~Hz}$, $1 \mathrm{H}) ;{ }^{13} \mathrm{C}$ NMR $\left(125 \mathrm{MHz}, \mathrm{CDCl}_{3}\right) \delta: 20.79,36.80,119.13$, $119.99,120.23,122.33,124.25,124.54,124.81,125.69$, $126.66,129.80,132.09,135.90,141.57,142.93,144.46$, 145.60, 147.33 .

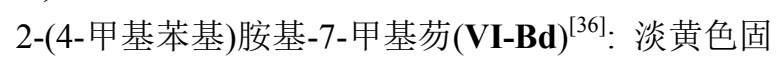
体, 产率 81.7\%. m.p. 139 $142{ }^{\circ} \mathrm{C} ;{ }^{1} \mathrm{H}$ NMR $\left(\mathrm{CDCl}_{3}\right.$, $400 \mathrm{MHz}) \delta: 2.31(\mathrm{~s}, 6 \mathrm{H}), 2.41(\mathrm{~s}, 3 \mathrm{H}), 3.74(\mathrm{~s}, 2 \mathrm{H})$, $6.99 \sim 7.05(\mathrm{~m}, 9 \mathrm{H}), 7.06(\mathrm{~d}, J=2.8 \mathrm{~Hz}, 1 \mathrm{H}), 7.13 \sim 7.22$ (m, 1H), 7.29 (s, 1H), $7.56(\mathrm{~d}, J=7.6 \mathrm{~Hz}, 2 \mathrm{H}) ;{ }^{13} \mathrm{C} \mathrm{NMR}$ $\left(125 \mathrm{MHz}, \mathrm{CDCl}_{3}\right) \delta: 20.78,21.57,36.65,118.88,119.89$, $120.24,122.46,124.13,124.48,125.59,127.48,129.76$, $131.95,135.47,138.93,143.23,144.29,145.67,146.87$.

\subsubsection{UV-vis 吸收光谱测定}

UV-vis 吸收光谱采用 Shimadzu UV-2450 测定, 记 录波长范围为 $300 \sim 600 \mathrm{~nm}$, 以 $\mathrm{CH}_{2} \mathrm{Cl}_{2}$ 为溶剂, $\mathrm{CH}_{2} \mathrm{Cl}_{2}$ 纯溶剂为基线, 高速扫描.

辅助材料(Supporting Information) 目标产物和中间 体化合物的 ${ }^{1} \mathrm{H}$ NMR 和 ${ }^{13} \mathrm{C} \mathrm{NMR}$ 图谱. 这些材料可以免 费从本刊网站(http://sioc-journal.cn/)上下载.

\section{References}

[1] Meng, H.; Zheng, J.; Lovinger, A. J.; Wang, B. C.; Van Patten, P. G.; Bao, Z. Chem. Mater. 2003, 15, 1778.

[2] Ahmed, S. A. M.; Al-Raqa, S. Y. J. Phys. Org. Chem. 2011, 24, 173.

[3] Higashijima, S.; Inoue, Y.; Miura, H.; Kubota, Y.; Funabiki, K.; Yoshida, T.; Matsui, M. RSC Adv. 2012, 2, 2721.

[4] Wu, Y.; Zhu, W. Chem. Soc. Rev. 2013, 42, 3453.

[5] Pei, J.; Liu, X. L.; Huang, W. Macromolecules 2003, 36, 323.

[6] Chen, X.; Tseng, H. E.; Liao, J.-L.; Chen, S.-A. J. Phys. Chem. B 2005, 109, 17496.

[7] Becker, K.; Lupton, J. M.; Feldmann, J.; Nehls, B. S.; Galbrecht, F.; Gao, D. Q.; Scherf, U. Adv. Funct. Mater. 2006, 16, 364.

[8] Lin, Y.; Chen, Y.; Gu, H. L.; Pan, Z.; Chen, J.-N. J. Funct. Polym. 2012, 25, 79 (in Chinese).

(林楹, 陈战, 顾慧丽, 潘喆, 陈军能, 功能高分子学报, 2012, 25,79 .)

[9] Jiao, S.-B.; Liao Y.; Xu, X.-J.; Wang, L.-P.; Yu, G.; Wang, L.-M.; Su, Z.-M.; Ye, S.-H.; Liu, Y.-Q. Adv. Funct. Mater. 2008, 18, 2335.

[10] Hao, Y.; Yang, X.-C.; Zhou, M.-Z.; Cong, J.-Y.; Wang, X.-N.; Hagfeldt, A.; Sun, L.-C. ChemSusChem 2011, 4, 1601.

[11] Haid, S.; Marszalek, M.; Mishra, A.; Wielopolski, M.; Teuscher, J.; Moser, J.-E.; Humphry-Baker, R.; Zakeeruddin, S. M.; Grätzel, M.; Bäuerle, P. Adv. Funct. Mater. 2012, 22, 1291.

[12] Cai, N.; Wang, Y.-L.; Xu, M.-F.; Fan, Y.; Li, R.-Z.; Zhang, M.; Wang, P. Adv. Funct. Mater. 2013, 23, 1846.

[13] Scrascia, A.; Marco, L. D.; Laricchia, S.; Picca, R. A.; Carlucci, C.; Fabiano, E.; Capodilupo, A. L.; Sala, F. D.; Gigli, G.; Ciccarella, G. J. Mater. Chem. A 2013, 1, 11909.

[14] Capodilupo, L. A.; Marco, L. D.; Fabiano, E.; Giannuzzi, R.; Scrascia, A.; Clarlucci, C.; Corrente, G. A.; Cipolla, M. P.; Gigli, G.; Ciccarella, G. J. Mater. Chem. A 2014, 2, 14181.

[15] Kobin, B.; Grubert, L.; Blumstengel, S.; Henneberger, F.; Hecht, S. J. Mater. Chem. 2012, 22, 4383.

[16] Song, Y.; Xu, W.; Zhu, D. Tetrahedron Lett. 2010, 51, 4894.

[17] Liu, T.-P.; Sun, J.; Li, R.; Tao, X.-C. Chin. J. Org. Chem. 2011, 31, 1799 (in Chinese). (刘涛平, 孙杰, 李瑞, 陶晓春, 有机化学, 2011, 31, 1799.)

[18] Kelkar, A.; Patil, M.; Chaudhari, R. V. Tetrahedron Lett. 2002, 43, 7143.

[19] Tsuji, J. Palladium Reagents and Catalysts, John Wiley \& Sons Ltd., Chichester, 2004, p. 289.

[20] Tao, X.-C.; Zhang, Y.-P.; He, T.-X.; Shen, D. Chin. J. Chem. 2007, $25,1326$.

[21] Chen, X.; Engle, K. M.; Wang, D.-H.; Yu, J.-Q. Angew. Chem. 2009, 121, 5196.

[22] Kakiuchi, F.; Chatani, N, Adv. Synth. Catal. 2003, 345, 1077.

[23] Hu, Q.-S. Synlett 2007, 1331.

[24] Liu, T.-P.; Xing, C.-H.; Hu, Q.-S. Angew. Chem., Int. Ed. 2010, 49, 2909. 
[25] Herrmann, W. A. Angew. Chem., Int. Ed. 2002, 41, 1290.

[26] Selvakumar, A.; Zapf, A.; Beller, M. Org. Lett. 2002, 4, 3031.

[27] Yang, C.; Lee, H. M.; Nolan, S. P. Org. Lett. 2001, 3, 1511.

[28] Hsiao, C.-C.; Lin, Y.-K.; Liu, C.-J.; Wu, T.-C.; Wu, Y.-T. $A d v$. Synth. Catal. 2010, 352, 3267.

[29] Chaumontet, M.; Piccardi, R.; Audic, N.; Hitce, J.; Peglion, J.-L.; Clot, E.; Baudoin, O. J. Am. Chem. Soc. 2008, 130, 15157.

[30] Qiu, Y.; Li, Y.-K.; Dong, H.; Duan, L.; Gao, Y.-D. CN 1931803, 2006 [Chem. Abstr. 2007, 146, 430917].

[31] Kang, J.-S.; Park, J. H.; Jun, S. W.; Shin, Y.-J.; Chang, Y.-M.; Yang, N.-C.; Park, J.-K.; Lee, S. WO 2014021572, 2014 [Chem. Abstr. 2014, 160, 319895].
[32] Li, X.-L.; Wu, W.; Fan, X.-H.; Yang, L.-M. RSC Adv., 2013, 3, 12091.

[33] Kang, J.-S.; Park, J. H.; Jun, S. W.; Shin, Y.-J.; Chang, Y.-M.; Yang, N.-C.; Park, J.-K.; Lee, S. WO 2014073791, 2014 [Chem. Abstr. 2014, 160, 714102].

[34] Wang, M.; Nalla, V.; Jeon, S.; Mamidala, V.; Ji, W.; Tan, L.-S.; Cooper, T.; Chiang, L.-Y. J. Phys. Chem. C Nanomater Interfaces 2011, 115, 18552.

[35] Ishida, T.; Mochizuki, F.; Yabuki, S.; Sawashita, Y.; Kurachi, M. JP 2005338446, 2005 [Chem. Abstr. 2005, 144, 14117].

[36] Senoo, A.; Yashiro, R.; Kikuchi, N.; Kanamaru, T. JP 03061952, 1989 [Chem. Abstr. 1991, 115, 170924]. 